\title{
HÁBITOS DE HIGIENE Y SEGURIDAD ALIMENTARIA EN UNA POBLACIÓN URBANA Y RURAL EN AISLAMIENTO POR COVID 19 REGIÓN TACNA, 2020
}

HYGIENE AND FOOD SAFETY HABITS IN AN URBAN AND RURAL POPULATION IN ISOLATION BY COVID 19 TACNA REGION, 2020

Gladys Eliana Zegarra Mandamiento ${ }^{1}$ Liz Mary Zelay Alanoca Chavez ${ }^{2}$ Raul Cartagena Cutipa ${ }^{3}$

Información del artículo:

Recibido: $27 / 10 / 2020$

Aceptado: $18 / 11 / 2020$

\footnotetext{
1,2 Escuela de Ingeniería agroindustrial, Universidad Privada de Tacna

${ }^{3}$ Docente Escuela de ingeniería agroindustrial, Universidad Privada de Tacna E-mail: ${ }^{1}$ gladys.zema@gmail.com, ${ }^{2}$ lizmaryzelay.ach@gmail.com, ${ }^{3}$ raucartagenac@upt.pe
} 


\section{Resumen}

La investigación tuvo como objetivo determinar los cambios en los hábitos de higiene y los impactos sobre la seguridad alimentaria en una población rural y urbana en aislamiento por Covid 19, en la región de Tacna. La investigación fue de tipo no experimental y transversal se usó la encuesta como técnica, aplicando un cuestionario a población con edades de 18 a 65 años, provenientes de provincias de Candarave representando a la zona rural y la provincia de Tacna clasificada como urbana. Para el análisis de datos se aplicó estadístico Chicuadrado de Pearson. Los resultados evidencian cambios significativos y positivos en los hábitos de higiene en la manipulación de alimentos que es más notoria en la zona urbana que en la zona rural, mientras que los impactos negativos sobre la seguridad alimentaria son más evidentes en la zona rural que en la zona urbana. Por lo tanto se concluye que hubo impactos significativos a consecuencia del confinamiento por Covid 19 sobre los hábitos de higiene y la seguridad alimentaria.

Palabras clave: Higiene alimentaria; seguridad alimentaria; Covid 19; aislamiento.

\section{Abstract}

The research aimed to identify changes in hygiene habits and impacts on food security in a rural and urban population in isolation by Covid 19 in the Tacna region. The research was non-experimental and crosscutting the survey was used as a technique, applying a questionnaire to populations aged 18 to 65 , from provinces of Candarave representing the rural area and the province of Tacna classified as urban. Pearson's Chisquare statistic was applied for data analysis. The results show significant and positive changes in hygiene habits in food handling that is more noticeable in urban areas than in rural areas, while negative impacts on food security are more evident in rural areas than in urban areas. It is therefore concluded that there were significant impacts as a result of Covid 19 confinement on hygiene habits and food safety.

Key words: Food hygiene; food safety: Covid 19; insulation. 


\section{Introducción}

Los alimentos son imprescindibles para toda la humanidad, pueden ser preparados y consumidos en casa o adquiridos en un establecimiento de venta, por ello la importancia de normas que regulan la higiene en la manipulación de alimentos, por otro lado la seguridad alimentaria es menos afín a poblaciones con menores recursos, esta situación se vive en muchas partes del mundo, empujando a los necesitados hacia una mala alimentación y padecer de hambre, en relación al número de personas con hambre (millones), la República Bolivariana de Venezuela $(6,8)$, Haití $(5,4)$, México $(4,7)$, Perú $(3,1)$, Guatemala $(2,6)$ concentraban más de la mitad de los subalimentados de la región según Sostenible \& FAO (2020), en ese contexto La crisis económica provocada por la pandemia del COVID 19 a nivel mundial, ha colocado en una difícil situación para poder equilibrar las restricciones que permitan en todo momento acceso físico, social y económico a cantidad de alimentos suficientes que sean inocuos y nutritivos para que puedan satisfacer las necesidades alimenticias y sus preferencias en cuanto a los alimentos con el fin de llevar una vida sana" (FAO, 2009). Es notorio que aun las repercusiones de la pandemia no se han detenido, muchas interrogantes se han planteado respecto de cuanto más se puede soportar los efectos del aislamiento y la falta de oportunidades que existe como consecuencia del COVID 19, Uno de los pilares afectados son directamente los sistemas alimentarios, mediante el impacto en la oferta y demanda de alimentos, también directa o indirectamente al poder adquisitivo y la capacidad de producir alimentos. La seguridad alimentaria ha sido sacudida, y aún no se ha superado esta crisis, dependerá mucho de las políticas que se implementen y el trabajo en equipo con los más vulnerables, principalmente en la post pandemia. Por otro lado, las condiciones y medidas que deben estar presentes en todas las etapas como producción, almacenamiento, transformación, transporte, conservación y cocinado doméstico del alimento deben garantizar la sanidad de los alimentos (Mundiales de la Salud, 201) también son importantes.

También es esa línea, los impactos son evidentes en Tacna; en sus inicios sobre el empleo de personas que perdieron el trabajo, producto de la paralización del comercio considerado como su principal motor económico, y tales aspectos podrían estar afectando positivamente en los hábitos de higiene y quizás negativamente en la seguridad alimentaria, principalmente en aspectos como el acceso a los alimentos (Klennert, 2005 y Schinca, 2009.), su disponibilidad (Solutions, 2013), uso y estabilidad (Clarke et al., 2015) pues en la actualidad existe mayor desconfianza cuando se adquiere un producto alimenticio fuera de casa, de tal forma que hay más exigencias en la higiene cuando se manipula alimentos. Sin embargo la incidencia de estos cambios podría ser distinta en la zona rural y urbana, por ello es importante conocer los efectos para planear formas de intervención no solamente del estado, sino de órganos que velan principalmente por la seguridad alimentaria. No menos importante es la higiene que debe tener el ciudadano. 


\section{Objetivos}

Determinar los cambios de hábitos de higiene en la manipulación de alimentos e impactos sobre la seguridad alimentaria en una población urbana y otra rural en estado aislamiento debido al Covid 19.

\section{Metodología}

La investigación es de tipo no experimental y transversal, en razón que las variables permiten medir los hábitos de higiene en la manipulación de alimentos e impactos sobre la seguridad alimentaria en una población urbana y rural en aislamiento por COVID 19. Tal como afirman Hernández et al., (2010), los diseños transaccionales descriptivos tienen como objetivo indagar la incidencia de las modalidades o niveles de una o más variables en una población.

Por tanto las variables a medir fue higiene alimentaria con 16 preguntas distribuidas en cuatro dimensiones; lavado de manos antes y durante la pandemia con nueve ítems, cocción de alimentos antes y durante la pandemia con cuatro ítems y agentes causantes de alimentos con tres ítems, mientras que la variable seguridad alimentaria se estructuró con 11 interrogantes que se distribuyeron en las dimensiones; disponibilidad de alimentos con tres ítems, acceso a alimentos con tres dimensiones, utilización con tres dimensiones, estabilidad con dos items.

El cuestionario se validó mediante el índice de fiabilidad de alfa de Crombach (79 \%), la muestra de estudio estuvo conformada por una población urbana y rural de la región de Tacna que se compone de dos provincias, la población rural representada por la provincia de Candarave y población urbana representada por la provincia de Tacna, para el cálculo de la muestra de estudio, se tomó como referencia la población reportada por INEI (2017) mediante "La Encuesta de Población y Vivienda efectuada el año 2017", para Tacna 212569 y Candarave 4055 habitantes, en consecuencia se calculó una muestra probabilística empleando la fórmula reportada por Hernández et al., (2010) resultando para Tacna 384 personas y Candarave 351 personas entre 18 y 65 años, se distribuyen proporcionalmente según sus distritos.

Para el análisis se agruparon a partir de las dimensiones según cada variable y se utilizó un contraste estadístico basado en el estadístico Chi-cuadrado.

\section{Resultados}

\section{Higiene Alimentaria}

Considerando el período crucial que el mundo vive actualmente a consecuencia de la pandemia Covid 19, es importante repensar en las medidas que la población debe asumir para hacer frente a eventos similares para mitigar los impactos, pensando que el virus se trasmite, entre otras vías por la oral, que puede estar asociado a un alimento que se ingiere. Por ello la necesidad de explorar los cambios que la población ha experimentado en el periodo de pandemia. Al explorar los cambios en la higiene alimentaria. 
La tabla 1 muestra el nivel de los cambios en hábitos de higiene en la manipulación de alimentos en aislamiento por Covid 19, se evidencia mayores cambio la provincia de Tacna (zona urbana) donde el mayor porcentaje de personas mostraron un alto nivel de cambios en sus hábitos de higiene en la manipulación de alimentos (83,1\%), de forma similar en la provincia de Candarave (zona rural) presentaron un alto nivel el $66,1 \%$. A través de la prueba Chi cuadrado de Pearson $\left(x^{2}=29,845\right)$ se determinó que existe relación significativa $(p$-valor $=0,000$ ) entre los niveles de cambio y la provincia de procedencia.

\section{Tabla 1}

Tabla de contingencia Nivel de higiene en la manipulación de alimentos

\begin{tabular}{|c|c|c|c|}
\hline \multirow{2}{*}{$\begin{array}{c}\text { Nivel de cambios higiene } \\
\text { en la manipulación de } \\
\text { alimentos }^{\mathrm{a}}\end{array}$} & \multicolumn{2}{|c|}{ Provincia ${ }^{b}$} & \multirow{2}{*}{ Total } \\
\hline & Candarave & Tacna & \\
\hline \multirow{2}{*}{ Bajo nivel } & 0 & 1 & 1 \\
\hline & $0,0 \%$ & $0,3 \%$ & $0,1 \%$ \\
\hline \multirow{2}{*}{ Moderado nivel } & 119 & 64 & 183 \\
\hline & $33,9 \%$ & $16,7 \%$ & $24,9 \%$ \\
\hline \multirow{2}{*}{ Alto nivel } & 232 & 319 & 551 \\
\hline & $66,1 \%$ & $83,1 \%$ & $75,0 \%$ \\
\hline \multirow[t]{2}{*}{ Total } & 351 & 384 & 735 \\
\hline & $100,0 \%$ & $100,0 \%$ & $100,0 \%$ \\
\hline
\end{tabular}

Nota: Elaboración propia. (a) y (b) están relacionados significativamente $\left(x^{2}=29,845, p\right.$ valor $=0,0001)$.

\section{Seguridad Alimentaria}

La seguridad alimentaria es el derecho que las personas tienen a disponer en todo momento de una suficiente cantidad y calidad de alimentos que le permitan una vida saludable y activa (ONU, 2014), por ello las restricciones impuestas por el gobierno pudieron haber tenido efectos sobre la disponibilidad y acceso, por ello la necesidad de analizar los efectos sobre la seguridad alimentaria, los resultados se muestra en la tabla 2 , donde se evidencia mayores efectos en la zona rural representada por Candarave $(73,4 \%)$ mientras que la zona urbana representada por Tacna (40\%) el impacto fue menor a causa de la pandemia. Mediante la prueba Chi cuadrado de Pearson $\left(x^{2}=64,800\right)$ se determinó que existe relación significativa ( $p$-valor=0.0001), entre los niveles de efectos sobre la seguridad alimentaria y el distrito. 


\section{Tabla 2}

Tabla de contingencia Nivel de seguridad alimentaria

\begin{tabular}{lccc}
\hline \multirow{2}{*}{ Nivel de afectacion } & \multicolumn{2}{c}{ Provincia $^{\mathrm{a}}$} & \multirow{2}{*}{ Total } \\
\cline { 2 - 3 } & Candarave & Tacna & \\
\hline Bajo Nivel & 0 & 1 & 1 \\
& $0,0 \%$ & $0,4 \%$ & $0,2 \%$ \\
\hline Moderado Nivel & 84 & 149 & 233 \\
& $26,6 \%$ & $59,6 \%$ & $41,2 \%$ \\
\hline Alto nivel & 232 & 100 & 332 \\
& $73,4 \%$ & $40,0 \%$ & $58,7 \%$ \\
\hline Total & 316 & 250 & 566 \\
& $100,0 \%$ & $100,0 \%$ & $100,0 \%$ \\
\hline
\end{tabular}

Nota: Elaboración propia. (a) y (b) están relacionados significativamente $\left(x^{2}=64,800, p\right.$-valor $\left.=0,0001\right)$.

\section{Discusiones}

\section{Higiene de alimentos}

Luego de la aparición de la pandemia, La alimentación en tiempos de confinamiento, tanto para la población no afectada por COVID-19 como para los afectados asintomáticos o con sintomatología leve, debe seguir los patrones de una alimentación saludable (CGCODN, 2020), por ello es importante que para asegurar alimentación saludable, se deba manejar higiénicamente todo alimento que se consume, dentro de este orden de ideas, se puede afirmar que la población de Tacna, mostro un nivel alto $(83,1 \%)$ de cambios en su higiene en el momento de manipularde alimentos, un porcentaje menor resulta para Candarave $(66,1 \%)$, ambos cambios son significativos, estos resultados serían evidencia que la población mejoró sus hábitos, que es atribuible a la llegada de la pandemia, que entre las acciones principales para evitar los contagios por coronavirus, la población recurrió al lavado de manos constante, usos de desinfectantes y empleo de protectores, estas acciones también fueron aplicadas en el higienización de los alimentos, no solo en los hogares, sino en los comercios donde se implementaron medidas precautorias desinfectando todo lo relacionado a la manipulación de alimentos (Eppens, 2020) esta posición es compartida por Gomes (2020) y Theumer (2020), quien indica que la población, especialmente los manipuladores de alimentos han implementado medidas de seguridad alimentaria.

\section{Seguridad alimentaria}

En la cadena de suministro de alimentos, esta pandemia ha llevado a los especialistas del área de ciencia y tecnología de alimentos a estudiar las distintas vías de propagación del virus y cómo evitar que los alimentos puedan ser una vía de contagio por contaminación cruzada (Romero et al., 2020), está claro que hay investigaciones que demuestran que no hay evidencias de que los alimentos sean fuentes 
o vías de trasmisión del coronavirus (Castell, 2020) este caso es afín seguridad alimentaria de las provincias de estudio, en particular de la rural, donde Los niveles altos de afectación sobre la seguridad alimentaria revelados en la provincia de Candarave $(73,4 \%)$, indicarían que la población tiene dificultades de disponer de la cantidad y calidad suficiente (ONU, 2014), y que al estar restringido la disponibilidad e inaccesibilidad a ellos estaría generando preocupación (Aulestia y Capa, 2020), otros agravantes es que al ser una población rural, donde el acceso fue limitado, y sumado a las deficiencias en las vías de comunicación y el disminución de ingresos económicos, habría agravado esta situación conforme a ello Rodríguez et al., (2020) indica que con la pandemia se vislumbra adverso y probablemente se exacerben las desigualdades alimentarias, por lo que la incertidumbre sobre la disponibilidad de alimentos debe conllevar a la implementación de medidas restrictivas para salvaguardar la seguridad alimentaria.

De lo expuesto se infiere que los tres pilares de la seguridad alimentaria se han visto afectadas, esta posición es compartida por Olivera et al., (2020), la explicación de estos efectos podría ser a causa de la disminución de ingresos, como afirma Viveros eta al., (2014) quien asevera que los indicadores socioeconómicos están asociados con el riesgo de inseguridad alimentaria, este aspecto es importante y frecuente en poblaciones rurales que tienen menor poder adquisitivo que a medida que se alarga la pandemia podrían presentar mayores efectos tal como concluye Herrera (2020) al investigar el empobrecimiento de los hogares y cambios en el abastecimiento de alimentos por la Covid-19 en lima, Perú, manifiesta las diferencias en el número de reducciones semanales de compras entre clases sociales, lo explicado incidiría en la calidad de alimentos que adquiere la población para suplir la cantidad habitual de consumo, tal como afirman Rojas y Mendieta (2020) quienes concluyen que a pesar que la población mantenga disponible la misma cantidad de alimentos, la disminución de ingresos tiende a agravar la calidad de alimentos que se consume en tiempos de pandemia, lo que puede llevar a otros problemas asociados, en esa dirección, Ayala (2020) indica que las medidas paliativas cobran importancia en el área rural donde los índices de pobreza son mayores por su parte Salle y Ayala (2020) concluyen que la crisis por COVID-19 está teniendo impactos únicos e individuales, desde la perspectiva del comportamiento alimentario se pueden considerar aquellas problemáticas asociadas a la seguridad alimentaria, así como a la selección y consumo de alimentos aspectos mediados no sólo por cuestiones económicas sino por el estado de ánimo y los cambios generados en el estilo de vida a nivel personal y social. En este sentido, la situación nutricional durante y posterior a la cuarentena resulta un aspecto prioritario. Como se manifiesta en la investigación, la pandemia está dejando impactos en aspectos asociados con la seguridad alimentaria.

Al analizar los efectos sobre la población urbana de Tacna se encontró que en su mayoría tuvo efectos moderados $(59,6 \%)$, siendo el nivel alto el 40,0 \%, a diferencia de la población rural de Candarave, las población urbana, al no presentar restricciones de acceso, y principalmente al contar con diversos centros de abasto donde pueden abastecerse de alimentos con normalidad, no parecen haber influido sobre la percepción de tener inseguridad alimentaria, así mismo otra explicación estaría en que la población urbana presentan mayores ingresos respecto a la rural, por lo que tendría mayores posibilidades de superar los efectos de la crisis asociado a la seguridad alimentaria por lo que el acceso, estabilidad y disponibilidad no habrían sido afectadas. 


\section{Conclusión}

Los efectos de la pandemia por Covid 19 en la higiene alimentaria en la población rural y urbana incidieron positiva y significativamente aumentando los niveles de higiene en la manipulación de alimentos en mayor porcentaje en la zona urbana que en la zona rural. El impacto en la seguridad alimentaria fue negativo, incidiendo en un nivel alto sobre la población rural, mientras que los efectos sobre la población urbana fueron moderados.

\section{Referencias Bibliográficas}

Academia Española de Nutrición y Dietética, Consejo General de Colegios Oficiales de DietistasNutricionistas (CGCODN) (2020). Recomendaciones de alimentación y nutrición para la población española ante la crisis sanitaria del COVID-19. Academia Española de Nutrición y Dietética, RedNube, El Consejo General de Colegios Oficiales de Dietistas-Nutricionistas; 2020. Disponible en: https://bit.ly/2WPLkIW.

Ayala Durán, C. (2020). Seguridad alimentaria y nutricional en tiempos de COVID-19: perspectivas para el Salvador. Disponible en https://n9.cl/ngqg

Aulestia-Guerrero, E. M., \& Capa-Mora, E. D. (2020). Una mirada hacia la inseguridad alimentaria sudamericana. Ciencia \& Saude Coletiva, 25(7), 2507-2517.

https://doi.org/10.1590/1413-81232020257.27622018

CEPAL, \& FAO. (2020). Comisión Económica para América Latina y el Caribe y Organización de las Naciones Unidas para la Alimentación y la Agricultura. Informe COVID-19: Cómo evitar que la crisis del COVID-19 se transforme en una crisis alimentaria. Acciones urgentes contra el h. 1-33.

Castell-Garralda, V. (2020). Seguridad alimentaria y Covid-19. Disponible en https://scientiasalut.gencat.cat/handle/11351/5004

Eppens, M. E. (2019). Higiene a la hora de comprar nuestros alimentos. Disponible en https://www.youtube.com/watch?v=ad6ocD0Ax10

Herrera Romero, T., 2020. Empobrecimiento de los hogares y cambios en el abastecimiento de alimentos por la Covid-19 en lima, PERÚ. http://hdl.handle.net/11086/15477

Instituto Nacional de Informática e Estadística. (2014). Encuesta Demográfica y de Salud Familiar-ENDES. $\begin{array}{llll}\text { Instituto Nacional de Estadística e Informática, } & 190 .\end{array}$ https://www.inei.gob.pe/media/MenuRecursivo/publicaciones_digitales/Est/Lib1211/pdf/Libro. pdf

Oliveira, T. C., Abranches, M. V., \& Lana, R. M. (2020). Food (in)security in Brazil in the context of the SARS-CoV-2 pandemic. Cadernos de Saude Publica, 36(4). https://doi.org/10.1590/0102$311 \times 00055220$

Ingeniería Investiga Vol. 2 (2) Julio - diciembre 2020 
Organización de las Naciones Unidas para la Alimentación y la Agricultura (2014). Indicadores de la seguridad alimentaria. Roma: FAO; 2014 [citado el 10 de noviembre de 2014]. Disponible en: http://goo.gl/ysbGa0

Rojas B., y Mendieta. E. M., (2020). Seguridad alimentaria en el Paraguay y Covid 19. Academic Disclosure, 1(1), 245-251. https://revistas.fenob.una.py/ojs/index.php/rfenob/article/view/93

Rodríguez Osiac, Lorena, Egaña Rojas, Daniel, Gálvez Espinoza, Patricia, Navarro-Rosenblatt, Deborah, Araya B, Marcela, Carroza, María Begoña, \& Baginsky G, Cecilia. (2020). Evitemos la inseguridad alimentaria en tiempos de COVID-19 en Chile. Revista chilena de nutrición, 47(3), 347-349. https://dx.doi.org/10.4067/S0717-75182020000300347

Romero, J., Agnetti, C., Coral, A., \& Medrano, A. (2020). RETOS EN LA CADENA DE SUMINISTRO DE ALIMENTOS ASOCIADOS A LA PANDEMIA DE COVID-19. Disponible en https://publitec.com/wpcontent/uploads/HPL-269_COVID.pdf

Salle, U. La, y Ayala-moreno, M. R. (2020). Cambios en el comportamiento alimentario en la era del COVID-19. Relais, 3(1), 27-30.

Schinca, N. (2009). El estado de la inseguridad alimentaria en el mundo. In Actividad Dietetica (Vol. 13, Issue 2). https://doi.org/10.1016/S1138-0322(09)71733-X

Solutions, H. P. (2013). En El Mundo. https://doi.org/10.5281/zenodo.583740

Sostenible, D., \& Fao, L. (2020). Seguridad Alimentaria bajo la Pandemia de COVID-19. Seguridad Alimentaria Bajo La Pandemia de COVID-19. https://doi.org/10.4060/ca8873es

Theumer, M. 2x1 (2020): medidas para evitar COVID-19 y enfermedades transmitidas por alimentos. Disponible en https://rdu.unc.edu.ar/handle/11086/16668 